\title{
Safety and efficacy of lobectomy combined with off-pump coronary artery bypass grafting for lung cancer
}

\author{
Zheng Wang ${ }^{1,2 \#}$, Fan Guo ${ }^{1,2 \#}$, Jiuzhen $\mathrm{Li}^{1,2}$, Daqiang Sun ${ }^{2}$ \\ ${ }^{1}$ Department of Graduate School, Tianjin Medical University, Tianjin, China; ${ }^{2}$ Department of Thoracic Surgery, Tianjin Chest Hospital, Tianjin, \\ China \\ Contributions: (I) Conception and design: D Sun, Z Wang; (II) Administrative support: D Sun; (III) Provision of study materials or patients: D Sun, Z \\ Wang; (IV) Collection and assembly of data: F Guo, J Li; (V) Data analysis and interpretation: Z Wang; (VI) Manuscript writing: All authors; (VII) \\ Final approval of manuscript: All authors. \\ "These authors contributed equally to this work. \\ Correspondence to: Daqiang Sun, MD. Department of Thoracic Surgery, Tianjin Chest Hospital, Tianjin Chest Hospital, No. 261 Taierzhuang South \\ Road, Jinnan District, Tianjin, China. Email: sdqmd@163.com.
}

Background: This study sought to assess the safety and efficacy of a lobectomy combined with off-pump
coronary artery bypass grafting (OPCABG) among patients with lung cancer.
Methods: Patients who underwent a simultaneous pulmonary lobectomy combined with OPCABG
at Tianjin Chest Hospital from December 2010 to June 2019 and patients who underwent pulmonary
lobectomy during the same period were selected at a ratio of $1: 2$ using a multi-factor propensity score for
this study. The clinical data of each group during the operation and perioperative period were analyzed and
compared, and the survival curves were drawn using the Kaplan-Meier method to compare the long-term
survival of the patients. Results: In the simultaneous-surgery group, no patients required a secondary thoracotomy for hemostasis and there were no perioperative deaths; however, 2 patients underwent a second intubation, 2 patients underwent a second debridement and suturing due to wound infection, 28 patients $(76.3 \%)$ had pulmonary complications, and 10 patients $(26.3 \%)$ had circulatory complications. The operation time, intraoperative blood loss, postoperative drainage volume, postoperative hospital stay, and complication rate were all higher in the simultaneous-surgery group than the lobectomy group. No significant difference was observed in the long-term survival rates between the 2 groups.

Conclusions: The simultaneous surgery was safe and effective. Some differences existed in the data between the 2 groups during the perioperative period; however, the risk of complications after surgery was controllable.

Keywords: Lobectomy; coronary artery bypass grafting; off-pump; simultaneous surgery

Submitted Apr 07, 2021. Accepted for publication Jun 17, 2021.

doi: $10.21037 /$ jtd-21-788

View this article at: https://dx.doi.org/10.21037/jtd-21-788

\section{Introduction}

According to global cancer statistics of 2018, the current incidence rate $(11.6 \%)$ and mortality rate $(18.6 \%)$ of lung cancer remains the highest among all cancers (1). In 2019, the Lancet analyzed the health models of 34 provincial administrative regions in China from 1990 to 2017, and found that premature death caused by coronary artery disease and lung cancer ranked second and third of all deaths respectively (2). Lung cancer and coronary heart disease have common pathogenic factors and susceptible populations of similar ages. As a result, the proportion of patients with non-small cell lung cancer (NSCLC) combined with coronary heart disease is gradually 


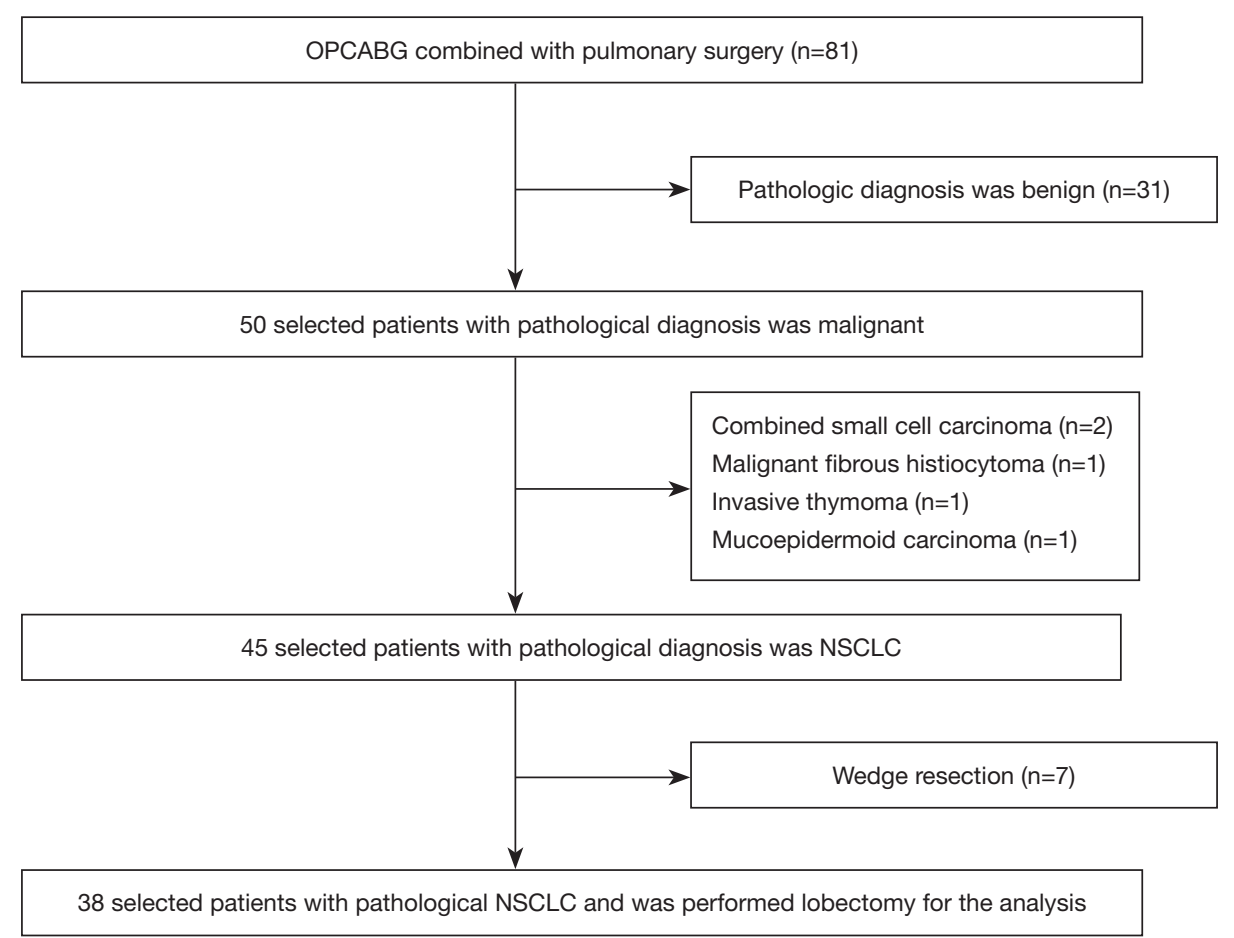

Figure 1 Flowchart depicting the patient selection process. OPCABG, off-pump coronary artery bypass grafting; NSCLC, non-small cell lung cancer.

increasing. Cardiopulmonary bypass (CPB) has been known to have transitory inhibitory effects on the components of the immune system including cell-mediated and humoral immunity. The off-pump procedure eliminates the various deleterious effects of CPB. In last decade, OPCABG has become more popular in clinical practice. Simultaneous surgery for these patients can reduce the risk of myocardial ischemia without delaying the treatment of NSCLC. In recent years, reports of simultaneous surgery have focused on the feasibility and short-term safety of the surgery; however, very few studies on the long-term survival of patients have been conducted. What is worse, previous studies have included several pathological types and the surgical approaches were varied, including wedge resection and pneumonectomy.

The present study examined 38 patients who underwent off-pump coronary artery bypass grafting (OPCABG) synchronized with a pulmonary lobectomy from December 2010 to June 2019 at Tianjin Chest Hospital. The clinical information of these patients was analyzed, and patients undergoing pulmonary lobectomy in the same period were matched via a multi-factor propensity score to compare the short-term safety and long-term survival of the simultaneous surgery.
We present the following article in accordance with the STROBE reporting checklist (available at https://dx.doi. org/10.21037/jtd-21-788).

\section{Methods}

This study reviewed the database of patients with lung cancer and coronary heart disease who received simultaneous surgery at Tianjin Chest Hospital, China from December 2010 to June 2019. On June 15, 2020, we first retrieved the names and hospital numbers of patients who had undergone a cardiac bypass combined lung surgery from the operating room registry. We then identified patients in the medical records room based on their names and medical records. From 2010 to 2019, a total of 81 patients, who had been diagnosed with coexistent coronary heart disease and lung neoplasm, underwent surgery at our hospital. A total of 38 patients with a pathologic diagnosis of NSCLC who underwent OPCABG combined with a pulmonary lobectomy were enrolled in Group A (see Figure 1). Patients who underwent an isolated lobectomy in the same time period were enrolled in Group B. The short-term safety and long-term survival of patients in the groups were 
Table 1 Patient baseline characteristics before and after propensity-score matching

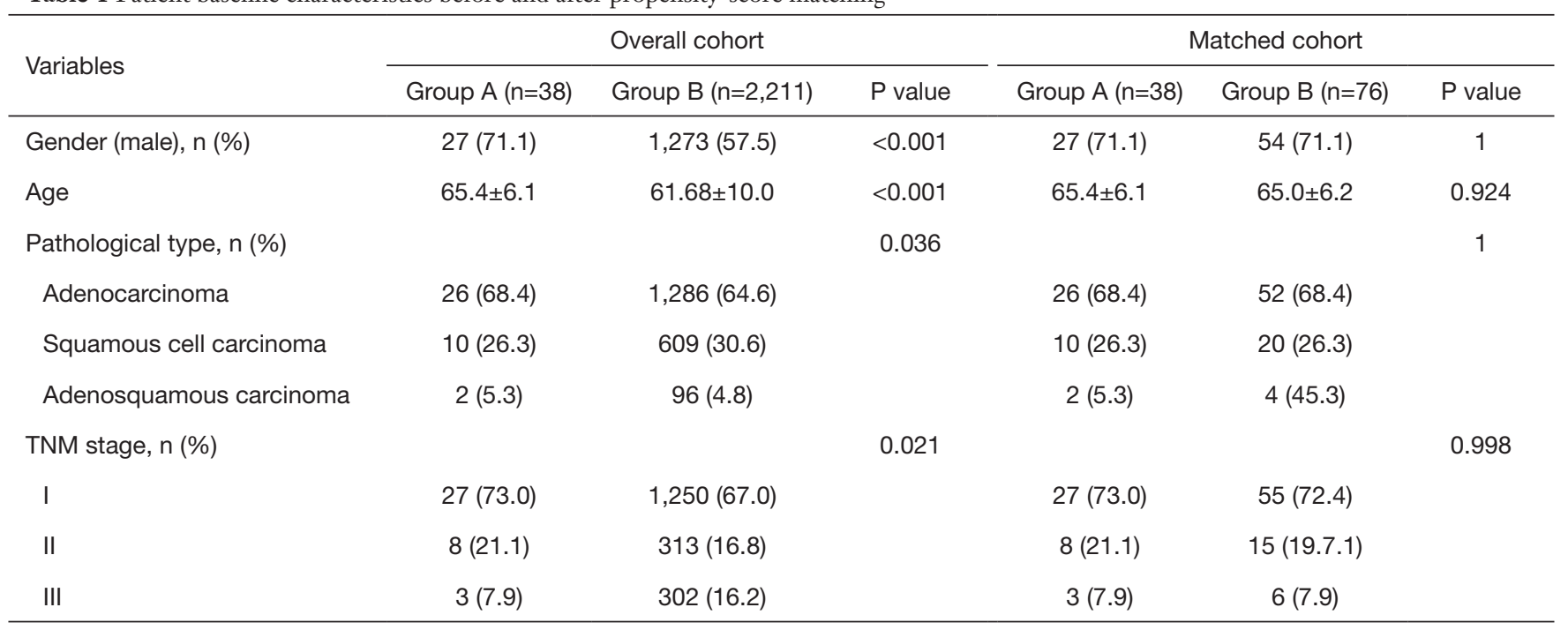

compared.

A propensity score-matched (PSM) analysis using Stata 11.2 was performed to balance any potential confounders between the groups. The propensity score was matched according to 4 factors (i.e., age, gender, tumor pathological type, and the tumor, nodes, and metastases (TNM) stage of the patients). Seventy-six patients who underwent pulmonary lobectomy during the same period were selected at a ratio of 1:2 and enrolled in Group B. The baseline clinical characteristics of the patients before and after PSM are set out in Table 1 .

The following clinical data were obtained from all patients: age, sex, pathological tumor characteristics, operation time, total estimated blood loss, time of intensive care unit (ICU) stay, time of hospital stay, incidence of complications and survival.

All procedures performed in this study involving human participants were in accordance with the Declaration of Helsinki (as revised in 2013). The study was approved by ethics committee of Tianjin Chest Hospital (No.2020LW-006: the registration number of ethics board) and informed consent was taken from all the patients.

\section{Operation procedure}

After double-lumen tube intubation and total intravenous anesthesia, the patients in Group A were first treated with an off-pump beating-heart coronary bypass operation. In the process of revascularization, if the patients failed to tolerate the off-pump beating-heart coronary bypass, a pulmonary lobectomy followed by a bypass operation after extracorporeal circulation was performed, and these patients were excluded from the study. According to the condition of the coronary artery, the great saphenous vein, left internal mammary artery or right radial artery of the lower extremity were taken for heparinization, the target vessel was fixed by the coronary artery fixator, the distal anastomosis was performed, and the proximal anastomosis was completed in the aorta under the aorta side-wall clamp. After hemostasis and heparin neutralization, a gauze pad was sewn on the right pericardium to cover the heart. After the patient's circulation was stabilized, a pulmonary lobectomy was performed in the same period by opening the pleura through the same incision or an auxiliary second incision. The decision of whether to use a thoracoscope to assist in debonding the lower pulmonary ligament, separating the pulmonary laceration, exposing the pulmonary artery, pulmonary vein, and bronchus, and detaching them with linear cutting suture unit was made on a case-by-case basis. After surgery, when a patient achieved complete hemostasis, a water test was carried out to dilate the lung and check for air leakage and bleeding, and the chest closure was made after the fixation of the drainage tube. The patient was returned to the ICU.

Patients in Group B underwent a routine pulmonary lobectomy and mediastinal lymph-node dissection.

After surgery, patients in Group A received a small dose of dopamine by continuous pumping in the initial stage, and a subcutaneous injection of low-molecular-weight heparin for anticoagulation was continued in the early stage. 
The general condition of the patients was evaluated. The patients were liberated from mechanical ventilation as early as possible to avoid postoperative pulmonary infection, and the patients transferred out of ICU were given oral anticoagulants. The patients were asked to get out of bed as early as possible to avoid the formation of deep vein thrombosis, and were given continuous atomization inhalation to promote cough and expectoration by turning over and slapping the back to avoid complications of pulmonary atelectasis and pulmonary infection. After surgery, the chest radiography was reexamined to observe for pneumothorax or pleural effusion. The discharge time was determined according to each patients' condition.

\section{Follow-up examinations}

Three, 6, and 12 months after surgery, patients' electrocardiographs, color-doppler echocardiography, and chest radiography scans were reexamined in the outpatient department, and their chest computed tomography (CT) scans were reexamined every 6 months. The patients mainly attended follow-up visits as outpatient or via telephone.

\section{Statistical analysis}

Excel software was used for the data clean-up, and SPSS 26.0 statistical software was used for data processing and analysis. The initial PSM analysis was conducted using STA software, and the continuous variables were tested for normal distribution. Variables conforming to the normal distribution were expressed as mean \pm standard deviation, and a $t$-test was used for comparison and analysis. Variables that did not conform to normal distributions were subjected to rank-sum tests. The classification variables were expressed as percentage compositions, and the comparison and analysis were carried out using the chi-square test. The survival curves were drawn using the Kaplan-Meier method, and were analyzed and compared using the Log-Rank test.

\section{Results}

\section{General information}

The reason for patients' visits was mostly discomfort in the precordium. A pulmonary nodule was identified by CT examination before surgery. The mean cardiac ejection fraction in Group A was $58.95 \% \pm 6.3 \%$; 1 patient had a grade I preoperative New York Heart Association cardiac function score, 29 patients had grade II, 8 patients had grade III. Seven patients had stable coronary heart disease, and 31 patients had unstable coronary heart disease. The cardiac ejection fraction, rate of hypertension complications, and prevalence of diabetes were worse in Group A than Group B. Table 2 compares the clinical data of Group A and Group B.

\section{Perioperative data}

The operation was completed successfully in both groups. In total, 114 patients required no secondary thoracotomy for hemostasis and survived the perioperative period of 90 days. The operation time $(268.3 \pm 53.4 v s .189 .8 \pm 35.2 \mathrm{~min}$; $\mathrm{P}<0.05)$, the postoperative observation time in ICU $(3.8 \pm 4.8$ vs. $2.2 \pm 0.4$ days; $\mathrm{P}<0.05)$, and the postoperative length of stay $(13.2 \pm 9.7$ vs. $9.4 \pm 3.3$ days; $\mathrm{P}<0.05)$ of Group A were all longer than those of Group B. The intraoperative blood loss $(585 \pm 273$ vs. $154.2 \pm 81.8 \mathrm{~mL} ; \mathrm{P}<0.05)$, the postoperative $24 \mathrm{~h}$ drainage volume $(850.4 \pm 228.4$ vs. $378.0 \pm 133.1 \mathrm{~mL}$; $\mathrm{P}<0.05)$, and the total drainage volume $(1,807 \pm 802.3$ vs. $1,095.1 \pm 273.8 \mathrm{~mL} ; \mathrm{P}<0.05)$ of Group A were all greater than those of Group B.

In Group A, 33 patients received median sternotomy surgery, 4 patients underwent a lateral thoracotomy and pulmonary lobectomy after median sternotomy bypass surgery, and 1 patient underwent coronary artery bypass grafting using the minimally invasive left thoracic incision approach, combined with a left superior lobectomy. During surgery, 4 patients were grafted with single bypass, 16 patients with a double bypass, 12 patients with a triple bypass, and 6 patients with a quadruple bypass. The great saphenous vein was used as the graft in 22 patients, the left internal mammary artery and the great saphenous vein were used as the grafts in 12 patients, the left internal mammary artery alone was used as the graft in 2 patients, and the right radial artery and the left internal mammary and the right radial artery were used as the grafts in 2 patients.

Most of the patients underwent intraoperative frozen pathological examinations, with the exception of some patients whose pathology results were obtained by bronchoscopy preoperatively. Subcarinal lymph nodes were thoroughly dissected in 13 patients, including 3 patients in stage IIIA and 10 patients in stage IA-IB. Systemic lymphnode dissections were performed for all patients in Group B.

Two patients in Group A underwent second intubation after extubation, and 2 patients received second debridement and suturing due to wound infection; 28 patients (76.3\%) experienced pulmonary complications, and these were 
Table 2 Demographic characteristics and comparison of clinical data

\begin{tabular}{|c|c|c|c|}
\hline Baseline characteristics & Group A (n=38) & Group B $(n=76)$ & $P$ value \\
\hline Ejection fraction, EF\% & $58.9 \pm 6.3$ & $63.6 \pm 3.2$ & $<0.001$ \\
\hline CAD & 38 & 21 & $<0.001$ \\
\hline Stable type, n (\%) & $7(18.4)$ & $21(100.0)$ & 0.281 \\
\hline \multicolumn{4}{|l|}{ NYHA level, n (\%) } \\
\hline I & $1(2.6)$ & - & \\
\hline II & $29(76.3)$ & - & \\
\hline III & $8(21.1)$ & - & \\
\hline Diabetes & $12(31.6)$ & $9(11.8)$ & 0.010 \\
\hline Cerebral infarction & $3(7.9)$ & $2(2.6)$ & 0.196 \\
\hline
\end{tabular}

CAD, coronary artery disease; EF, ejection fraction; NYHA, New York Heart Association.

significantly higher in Group A than Group B (10 patients, $13.2 \%)$. Specifically, 28 patients suffered from pulmonary infections, 3 from pleural effusions, and 1 from atelectasis; 10 patients $(26.3 \%)$ had circulatory complications, of whom 3 had atrial fibrillations, 4 had cardiac insufficiency, 1 had a myocardial infarction, 1 had a cardiac arrest, and 1 had a cerebral infarction. A total of 29 patients (76.3\%) developed perioperative complications. Comparisons of operative and perioperative data are shown in Table 3.

\section{Follow-up data}

Thirty-eight patients in Group A were followed up by telephone for 5-98 months. The follow-up rate of Group A was $100 \%$. Four patients died. Of these 4 patients, 3 died of lung cancer recurrence, and 1 of myocardial infarction. In addition, 1 patient with brain metastases is now living with a tumor. The follow-up time of Group B was 6.5-101 months with a follow-up rate of $97.4 \%$. Of the patients in Group B, 15 patients died of lung cancer recurrence. The 3-year overall survival rates of patients in Group A and Group B were $93.6 \%$ and $82.7 \%(\mathrm{P}=0.168)$, respectively (see Figure 2$)$.

\section{Discussion}

As a result of progress in diagnostic techniques and improvements in group health awareness, the number of patients diagnosed with lung cancer and coronary heart disease in China is increasing. Despite a multitude of medical strategies, including chemotherapy, radiotherapy, targeted therapy, and immunotherapy, surgery is still the first choice for patients with resectable NSCLC (3). According to preoperative evaluations, medical interventions or surgical treatments are required by $5 \%$ of patients awaiting lung cancer surgery (4). Percutaneous coronary intervention (PCI) and bypass surgery have their respective indications (5); however, the standard anticoagulation therapy required after PCI and the postoperative recovery period of coronary artery bypass surgery both delay the treatment time of NSCLC. The incremental risk of noncardiac surgery on adverse cardiac events among poststent patients is highest in the initial 6 months following stent implantation, and stabilizes at $1.0 \%$ after 6 months. Elective, high-risk, inpatient surgery, and patients with drug-eluting stents may benefit the most from a 6-month delay after stent placement (6).

The guidelines developed jointly by the American College of Cardiology and American Heart Association recommend that patients with drug-eluting stent implants undergo non-cardiac surgery at least 1 year later, as the early discontinuation of antiplatelet drugs may increase the risk of stent thrombosis. If the risk of delayed surgery is greater 
Table 3 Comparison of operative and perioperative data

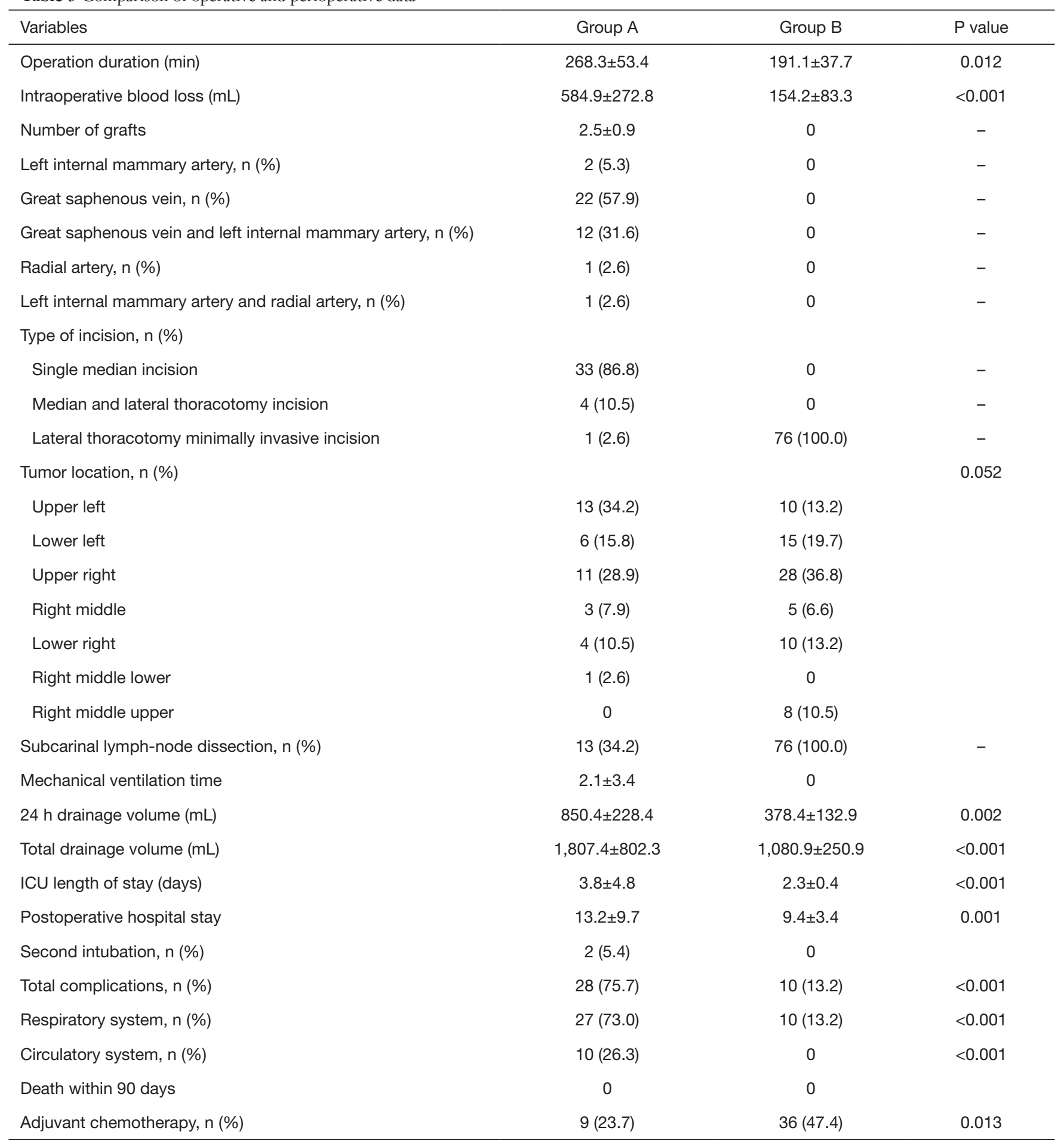

than the risk of expected ischemia or stent thrombosis after the implantation of a drug-eluting stent, the non-cardiac surgery may be delayed by 180 days (7). Simultaneous surgery avoids the delay of lung cancer surgery and reduces the trauma caused by 2 operations, the length of stay and costs; however, it also presents greater challenges for surgeons. Previous studies have mostly been retrospective studies of single-centers with small sample sizes and have 


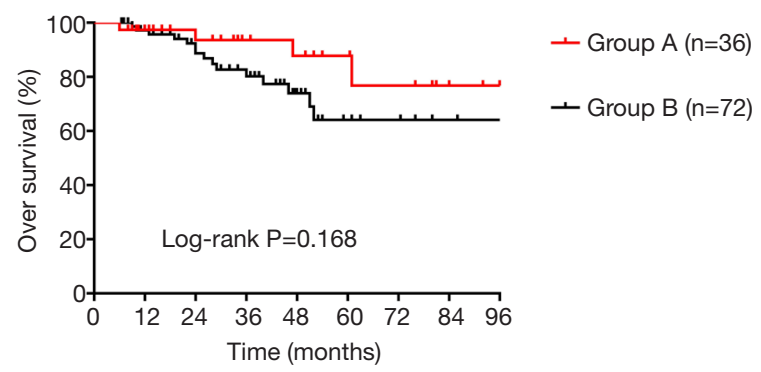

Figure 2 Comparison of Kaplan-Meier survival curves between the 2 groups.

included all types of lesions (e.g., small cell lung cancer, thymoma, bronchoalveolar carcinoma, and even benign lesions). The surgical approaches were several patterns, including wedge resection and pneumonectomy (8-11). The above-mentioned factors may be confounders that interfere with the outcomes. The present study represents the largest retrospective analysis of NSCLC patients who have undergone OPCABG and a lobectomy simultaneously at a single center. In our experience, the simultaneous surgery was safe and effective, and the risk of complications after the operation was controllable.

In terms of operation sequence, several articles have proposed that OPCABG should be performed before lung cancer radical operation for 2 reasons (8). First, to minimize adverse events during lung cancer radical operation to ensure myocardial blood supply. Second, as coronary artery bypass grafting produces a type 1 incision and pulmonary tumor resection produces a type 2 incision, the aseptic environment of mediastinum will be destroyed if the pleura is opened. As the consequences of chest infections are very serious, it is necessary to strictly ensure the sterility of surgical operations, and perform radical operations after bypass surgery. We also agree with the view that OPCABG should be performed before the radical resection of lung cancer (as occurred in this group of 38 patients).

The standard approach for coronary bypass surgery uses a mid-sternal incision. OPCABG has also been performed using a left anterior outboard incision, but the procedure is suitable for the anastomosis of a single-vessel disease with the left anterior descending branch. The approach for the radical resection of lung cancer depends on the growth site of the tumor; however, the lateral thoracotomy approach is generally adopted. In the early stage, the doctors who performed this operation mostly adopted the doubleincision approach; that is, they performed the coronary artery bypass grafting through the median sternotomy, and then transferred the lateral thoracotomy to open the pleura for the radical resection of lung cancer. However, the double-incision approach has many complications and results in strong postoperative pain, which may even affect the postoperative cough and expectoration of patients, and is prone to lead to postoperative pulmonary complications, such as atelectasis and pulmonary infection. According to reports, the radical resection of lung cancer can also be safely performed via a mid-sternal incision (12). In recent years, most surgeons have performed the simultaneous surgery via the same incision $(8,10)$. The disadvantage of the mid-sternal incision is that the hilar tissue is not exposed as clearly as that in the lateral thoracotomy in tumors located in the left lung, especially in the left lower lobe, which results in certain interference in the subsequent dissection of mediastinal lymph nodes. Of the 38 patients in Group A, 33 patients received median sternotomy surgery, 4 patients underwent a lateral thoracotomy and pulmonary lobectomy after median sternotomy bypass surgery, and 1 patient underwent coronary artery bypass grafting with a minimally invasive incision via the left thoracic approach, combined with a left superior lobectomy.

Mediastinal lymph-node metastasis is an important factor affecting the prognosis of patients with NSCLC (13). As mentioned above, for tumors located in the left lower lobe of the lung, if a pulmonary lobectomy is performed using the median sternal approach, it is difficult to expose the hilar structure, as the heart is in the left anterior position, which results in greater difficulties in the subsequent dissection of the subcarinal lymph nodes. A larger lymph-node dissection area is bound to cause greater surgical trauma, especially if there is poor exposure and anatomical variation near the carina, and it is very easy to cause postoperative complications (14). To ensure the safety of patients and reduce intraoperative complications, subcarinal lymphnode dissection was abandoned in 25 patients. Compared with the matched group, no significant difference in longterm survival was observed between the 2 groups. Thus, the subcarinal lymph nodes could be selectively sampled in the simultaneous-surgery of patients with early lung cancer (as confirmed by preoperative assessments). However, due to the small sample size of this group, further research needs to be conducted on lymph-node dissection methods under difficult exposure conditions to minimize trauma and optimize survival prognosis.

In terms of perioperative safety, the incidence of postoperative complications in Group A was significantly 
higher than that in Group B for 2 reasons. First, combining a 2-stage surgery into a 1-stage surgery causes more trauma among patients. Second, as coronary artery bypass grafting requires a type 1 incision and lung-tumor resection requires a type- 2 incision, simultaneous surgery may increase the incidence of infection-related complications. In the present study, there were no mediastinal infections, and only 2 patients had sternum-related infections that were treated by secondary management. Thus, no significant increase in the risk of infection was found. As previous studies have reported, the incidence of respiratory complications of combined surgery increased significantly compared to isolated surgery.

The authors believe that the outcomes may be related to the distribution of departments at our Center, at which the Thoracic Department and Cardiac Department are located in 2 sections. If the risk of a cardiac event is higher than a pulmonary event, patients are hospitalized in the Cardiac Department, and a thoracic surgeon is consulted if necessary. A lack of postoperative attention to the lungs led to a high incidence of respiratory complications . Similar to the results of previous studies, circulatory complications occurred in 9 patients in the present study. The average postoperative hospitalization period for the 38 patients in Group A was 13.2 \pm 9.7 days; the longest postoperative hospitalization period was up to 50 days. The complication rate was higher in the simultaneous-surgery group than the single-disease surgery group; however, the risk was controllable, and patients were cured and discharged after active treatment. Sub-optimal outcomes may occur perioperatively; however, this can be addressed through the optimization of postoperative treatment procedures and progressive technical developments. We look forward to publishing more desirable results for future studies.

According to our observations, the number of patients receiving postoperative adjuvant chemotherapy after simultaneous surgery was less than that of patients receiving single-disease surgery for 2 reasons. First, the trauma caused by simultaneous surgery was relatively large, resulting in a long postoperative recovery time for the patients, who also could not bear the side effects of postoperative chemotherapy within a short period. Second, while the risk of cardiotoxicity in association with chemotherapy in patients with lung cancer has been extensively studied (15), the long-term effects of chemotherapy on vein bypass grafts remains unclear. The 3 -year survival rates were $93.6 \%$ and $82.7 \%$ for patients who underwent simultaneous surgery and single-disease surgery, respectively. The long-term survival rate of patients with lung cancer and coronary heart disease was not reduced by the simultaneous surgery.

The following should be noted during the operation: (I) protect the myocardial bridge and reduce the compression and traction of heart and lung tissues; (II) the order of convenience for hilar exposure is: upper lobe > lower lobe, right lung > left lung; (III) the upper lobe is easier to remove in single-direction lobectomy. When removing the lower lobe, the inferior pulmonary ligament should be loosened and lower pulmonary veins cut off first, to better expose the inferior branch of the pulmonary artery through the interlobar fissure and ensure safe and convenient handling of arteries and blood vessels; (IV) when exposing the left pulmonary artery after midline laparotomy, it will be blocked by the left bronchus, while when exposing the right bronchus it will be blocked by the right pulmonary artery. Therefore, the relationship between the trunk and branch should be noted to avoid accidental injury; (V) the use of endovascular gastrointestinal anastomosis stapler makes the operation more convenient and also reduces the risk of bleeding; (VI) midline laparotomy could be performed through the front of the hilum to expose the subcarinal lymph nodes, especially after the upper lobe removal which is more convenient; (VII) adequate hemostasis before the end of the operation could avoid and reduce the risk of another thoracotomy to stop bleeding, and early anticoagulation will not increase the risk of another thoracotomy to stop bleeding

The PSM process can reduce potential biases in retrospective studies; however, unlike randomized controlled trials, the biases caused by unobserved covariates cannot be eliminated. To our knowledge, this study is the largest retrospective analysis of NSCLC patients who have undergone OPCABG and a lobectomy simultaneously at a single center. We hope that in the future, more studies, particularly large, randomized prospective studies, will focus on this topic to further verify our results.

\section{Conclusions}

To conclude, some differences in the perioperative data between the simultaneous-surgery and the single-disease surgery groups were observed; however, all patients were discharged safely after receiving individual treatment. Further studies need to be conducted to optimize the simultaneous-surgical procedures and minimize the 
perioperative complications.

\section{Acknowledgments}

Funding: None.

\section{Footnote}

Reporting Checklist: The authors have completed the STROBE reporting checklist. Available at https://dx.doi. org/10.21037/jtd-21-788

Data Sharing Statement: Available at https://dx.doi. org/10.21037/jtd-21-788

Conflicts of Interest: All authors have completed the ICMJE uniform disclosure form (available at https://dx.doi. org/10.21037/jtd-21-788). The authors have no conflicts of interest to declare.

Ethical Statement: The authors are accountable for all aspects of the work in ensuring that questions related to the accuracy or integrity of any part of the work are appropriately investigated and resolved. All procedures performed in this study involving human participants were in accordance with the Declaration of Helsinki (as revised in 2013). The study was approved by ethics committee of Tianjin Chest Hospital (No.2020LW-006: the registration number of ethics board) and informed consent was taken from all the patients.

Open Access Statement: This is an Open Access article distributed in accordance with the Creative Commons Attribution-NonCommercial-NoDerivs 4.0 International License (CC BY-NC-ND 4.0), which permits the noncommercial replication and distribution of the article with the strict proviso that no changes or edits are made and the original work is properly cited (including links to both the formal publication through the relevant DOI and the license). See: https://creativecommons.org/licenses/by-nc-nd/4.0/.

\section{References}

1. Bray F, Ferlay J, Soerjomataram I, et al. Global cancer statistics 2018: GLOBOCAN estimates of incidence and mortality worldwide for 36 cancers in 185 countries. CA Cancer J Clin 2018;68:394-424. Erratum in: CA Cancer J Clin 2020;70:313.
2. Zhou M, Wang H, Zeng X, et al. Mortality, morbidity, and risk factors in China and its provinces, 1990-2017: a systematic analysis for the Global Burden of Disease Study 2017. Lancet 2019;394:1145-58. Epub 2019 Jun 24. Erratum in: Lancet 2020;396:26.

3. Mei J, Guo C, Xia L, et al. Long-term survival outcomes of video-assisted thoracic surgery lobectomy for stage I-II non-small cell lung cancer are more favorable than thoracotomy: a propensity score-matched analysis from a high-volume center in China. Transl Lung Cancer Res 2019;8:155-66.

4. Dyszkiewicz W, Jemielity MM, Piwkowski CT, et al. Simultaneous lung resection for cancer and myocardial revascularization without cardiopulmonary bypass (offpump coronary artery bypass grafting). Ann Thorac Surg 2004;77:1023-7.

5. Jones DW, Minhas S, Fierro JJ, et al. From WOEST to AUGUSTUS: a review of safety and efficacy of triple versus dual antithrombotic regimens in patients with atrial fibrillation requiring percutaneous coronary intervention for acute coronary syndrome. Ann Transl Med 2019;7:405.

6. Holcomb CN, Graham LA, Richman JS, et al. The incremental risk of noncardiac surgery on adverse cardiac events following coronary stenting. J Am Coll Cardiol 2014;64:2730-9.

7. Fleisher LA, Fleischmann KE, Auerbach AD, et al. 2014 ACC/AHA guideline on perioperative cardiovascular evaluation and management of patients undergoing noncardiac surgery: executive summary: a report of the American College of Cardiology/American Heart Association Task Force on Practice Guidelines. Circulation 2014;130:2215-45.

8. Li Z, Liu B, Ge W, et al. Effect of simultaneous surgical treatment of severe coronary artery disease and lung cancer. J Int Med Res 2019;47:591-9.

9. Liu B, Chen C, Gu C, et al. Combined Coronary Artery Bypass Graft (CABG) Surgery and Lung Resection for Lung Cancer in Patients More than 50 Years-of-Age. Med Sci Monit 2018;24:3307-14.

10. Yeginsu A, Vayvada M, Karademir BC, et al. Combined Off-Pump Coronary Artery Bypass Grafting and Lung Resection in Patients with Lung Cancer Accompanied by Coronary Artery Disease. Braz J Cardiovasc Surg 2018;33:483-9.

11. Dyszkiewicz W, Jemielity M, Piwkowski C, et al. The early and late results of combined off-pump coronary artery bypass grafting and pulmonary resection in patients with concomitant lung cancer and unstable coronary heart 
disease. Eur J Cardiothorac Surg 2008;34:531-5.

12. Urschel HC Jr, Razzuk MA. Median sternotomy as a standard approach for pulmonary resection. Ann Thorac Surg 1986;41:130-4.

13. Izbicki JR, Thetter $O$, Habekost $M$, et al. Radical systematic mediastinal lymphadenectomy in non-small cell lung cancer: a randomized controlled trial. Br J Surg 1994;81:229-35.

14. Alam N, Park BJ, Wilton A, et al. Incidence and risk

Cite this article as: Wang Z, Guo F, Li J, Sun D. Safety and efficacy of lobectomy combined with off-pump coronary artery bypass grafting for lung cancer. J Thorac Dis 2021;13(7):44384447. doi: $10.21037 /$ jtd-21-788 factors for lung injury after lung cancer resection. Ann Thorac Surg 2007;84:1085-91; discussion 1091.

15. Hardy D, Liu CC, Cormier JN, et al. Cardiac toxicity in association with chemotherapy and radiation therapy in a large cohort of older patients with non-small-cell lung cancer. Ann Oncol 2010;21:1825-33.

(English Language Editor: L. Huleatt) 